\title{
Enquête sur un écrit de savoir au lycée : la dissertation littéraire
}

\section{François Le Goff}

\section{Q OpenEdition}

Journals

\section{Édition électronique}

URL : http://journals.openedition.org/pratiques/1409

DOI : 10.4000/pratiques. 1409

ISSN : 2425-2042

\section{Éditeur}

Centre de recherche sur les médiations (CREM)

\section{Édition imprimée}

Date de publication : 15 décembre 2009

Pagination : 111-126

\section{Référence électronique}

François Le Goff, «Enquête sur un écrit de savoir au lycée : la dissertation littéraire », Pratiques [En ligne], 143-144 | 2009, mis en ligne le 13 juin 2014, consulté le 19 avril 2019. URL : http:// journals.openedition.org/pratiques/1409; DOI : 10.4000/pratiques.1409 


\title{
Enquête sur un écrit de savoir au lycée : la dissertation littéraire
}

\author{
François Le Goff \\ IUFM Midi-Pyrénées, UT2, ÉA 4152
}

\section{Introduction}

En 1990, la revue Pratiques consacre un numéro à l'exercice de la dissertation (décembre 1990, n68). D'autres numéros ont depuis lors proposé l'examen d'une question spécifique comme la recherche des idées (Delforce, 1992), l'apprentissage (Darras, 1992), l'exemplification (Delcambre, 1996). Des publications faisant état de travaux didactiques ou proposant des démarches d'apprentissage ont paru en nombre au cours des années 90 (Preiss, 1989 ; Scheiber, 1989 ; Darras, 1994 ; Colson, 1996 ; Thyrion, 1996 ; Adam, 1999) mais il faut bien reconnaître que depuis l'essor des études consacrées à la lecture et à la production des discours argumentatifs, l'intérêt pour la dissertation a nettement faibli. Bon ou mauvais signe ? Comment est perçue la dissertation au lycée par ceux-là mêmes qui l'enseignent et l'évaluent? Quels jugements les professeurs portent-ils sur son apprentissage et son rôle dans l'enseignement des savoirs littéraires?

Pour tenter d'apporter quelques réponses à ces questions, j'ai adopté le format de l'enquête, déjà retenu il y a quatre ans pour interroger les pratiques de classe de l'écriture d'invention, exercice introduit au lycée au moment de la réforme des programmes de 2001 (Le Goff, 2005). Certes, les précautions d'usage s'imposent; ce sont des pratiques déclarées, des discours reçus à une échelle modeste puisque je me fonde sur l'analyse de 82 questionnaires, originaires d'une même région, Midi-Pyrénées. Et l'on sait par ailleurs la distance qu'il peut exister entre une rapide synthèse factuelle, un jugement de valeur et la réalité qu'ils sont censés décrire. Sans ignorer ces réserves, il m'a semblé intéressant pour les professeurs eux-mêmes, pour les différents acteurs de la formation, et plus largement pour la réflexion didactique, de recueillir la parole de l'enseignant. Signalons enfin et sans entrer plus avant dans les détails de l'analyse que la confrontation des réponses fournies permet de dégager des axes de lecture assez nets et suffisamment cohérents pour que leur soit accordée quelque valeur.

Au chapitre des informations méthodologiques, je précise que le recueil des données s'est déroulé au cours du premier semestre 2009. Plus de 25 établissements ont été contactés, 82 questionnaires ont été retournés, tous se sont avérés exploitables. L'examen statistique a été mené à partir du logiciel Microsoft Excel, 
les renseignements quantitatifs ont fait l'objet d'une analyse et d'un commentaire synthétiques. La présentation des résultats de l'enquête telle qu'elle apparaît dans cet article ne reproduit pas l'ordre du questionnaire. Je me suis en effet appuyé sur les réponses et les orientations qu'elles dessinaient pour bâtir l'exposé et définir le fil de sa démonstration.

\section{La défense d'un exercice critiqué}

Lorsque Michel Charolles, en 1990, intitule son article « La dissertation quand même » (Charolles, 1990) et prend soin de souligner le caractère collectif de l'entreprise, on imagine sans peine que le sujet est polémique, inspire des réticences, voire plus. Peu de temps auparavant, la rédaction, pratique scolaire du collège a déjà été remise en cause fortement dans ses principes et ses applications (Halté, 1989) et la dissertation pourrait aussi recevoir des critiques de même nature. A un moment où la discipline « français » se recompose, notamment autour des théories du texte et des typologies textuelles, où l'argumentation devient un objet d'enseignement structuré, étayé théoriquement et modifie les pratiques de lecture et d'écriture, où les productions écrites se développent en référence à des discours sociaux de référence, la dissertation apparaît alors bien suspecte et représente une forme d'évaluation de l'enseignement de la littérature quelque peu artificielle, marquée trop fortement par son empreinte rhétorique. Certes, on ne parle plus alors de dissertation (le terme a été remplacé dès 1969) mais de « composition française », de " discussion ». Cependant, les cadres formels de production demeurent alors même que les outils de description et formalisation des discours argumentatifs font l'objet d'un large mouvement de transposition didactique et tendent à privilégier des formes rédactionnelles présentant des situations énonciatives plus authentiques. Il est en outre exact que l'exercice est retenu par un nombre très faible de candidats aux épreuves anticipées de français (désormais EAF), qu'il suppose une solide culture littéraire que « les nouveaux lycéens » n'ont pas, qu'il s'apparente bien souvent à une addition de considérations convenues et stéréotypées, très éloignées de ce que pourrait être l'expression d'une expérience sensible et personnelle des textes littéraires.

Pourtant, quelques années plus tard, l'exercice a retrouvé son appellation d'origine (MEN, 2001a) ; les renseignements pris auprès de correcteurs des EAF laissent supposer que l'épreuve recueille plus de suffrages qu'il y a vingt ans. La dissertation retrouverait-elle une valeur d'estime? Si l'on en juge par la réponse opposée à l'affirmation 10 du questionnaire, on observe que les professeurs de français sont très attachés à cet exercice. En effet, la possibilité d'une suppression est rejetée fermement par près de $80 \%$ des opinions exprimées. Seuls $4 \%$ des professeurs seraient d'un avis contraire mais sans que cela soit fortement défendu. Il semble bien que la dissertation ait trouvé de nouveaux défenseurs après des années de discrédit. Cette hypothèse est renforcée par les réponses à 1 'item 11. La place de la dissertation dans leur propre enseignement est jugée « importante» par les professeurs pour près de $40 \%$ d'entre eux $(38 \%)$ et $«$ essentielle » pour $9 \%$ d'entre eux. Les commentaires qui accompa-

\begin{tabular}{|c|c|}
\hline $\begin{array}{c}\text { valeur } \\
\text { manquante }\end{array}$ & $\begin{array}{c}\text { tout à fait } \\
\text { d'accord }\end{array}$ \\
plutôt \\
d'accord \\
ni d'accord \\
ni pas \\
d'accord \\
$2 \%$ \\
pas du tout \\
d'accord \\
$78 \%$ \\
Fig 10: La dissertation pourrait être \\
supprimée de la classe de français \\
\hline
\end{tabular}


gnent certaines des réponses expliquent l'introduction d'une autre entrée nommée « dépendante $»$. En effet, dans $11 \%$ des réponses, il est précisé que la place de la dissertation varie en fonction des niveaux de classe et de leurs profils ainsi que des enseignements qui sont privilégiés en cours d'année.

En tant qu'écrit de savoirs, après plus d'un siècle d'existence (Jey, 1998) et en dépit des désaffections qu'elle a pu connaître, la dissertation fait à l'évidence partie de ces objets qui dessinent les contours de la discipline « français » au lycée. Sa suppression équivaudrait à amputer la discipline d'un de ses piliers et à dénaturer les enseignements littéraires. Elle rejoint ici le commentaire littéraire dans la fondation de l'identité de la discipline. Cette épreuve très française, inconnue hors de nos frontières (Wlassoff, 1998) fait peut-être l'objet d'un attachement d'ordre affectif de la part de l'enseignant puisque cet exercice a jalonné sa formation universitaire et a sanctionné sa réussite aux concours de recrutement de l'Éducation nationale. Mais il y a bien sûr des motivations plus objectives, plus professionnelles qui justifient cet intérêt. Et c'est parce que cet attachement apparaît si marqué dans les enquêtes dont je dispose et que la proportion d'avis favorables au maintien de la dissertation m'a surpris que j'ai retenu cette donnée comme fil directeur de l'analyse. Quels sont les motifs de cet attachement, et comment celui-ci influence-t-il les apprentissages et l'évaluation de l'exercice?

\section{Pas de dissertation sans savoirs}

L'histoire de la dissertation est étroitement associée à celle de la littérature et de son enseignement. A la fin du XIX ${ }^{\mathrm{e}}$ siècle, le passage d'un enseignement rhétorique fondé sur une imitation des modèles à un examen critique des formes et des manifestations littéraires consacre la dissertation ; elle devient progressivement la référence en matière de formalisation des discours scolaires sur la littérature. (Houdart-Merot, 1998 ; Rey, 1998 ; Viala, 1990). Les connaissances littéraires déclinées en genres et registres, en mouvements littéraires, distribuées dans une chronologie, mises au jour et discutées dans le temps des lectures d'œuvres intégrales ou de fragments sont susceptibles d'être reconfigurées dans des discours qui ont pour fonction de stabiliser un savoir récemment acquis et de le mettre en perspective avec d'autres savoirs. Au côté notamment de la synthèse ou de l'exposé mais avec d'autres ambitions, la dissertation participe à la mise en forme, à la recomposition des connaissances. En tant qu'écrit de savoir, elle reconfigure des faits, les oriente et les met en dialogue dans la perspective d'une démonstration. Par le jeu de l'interrogation qu'elle propose en subordonnant les savoirs au filtre d'une problématique, il y a cette idée que le savoir est vivant. En d'autres termes, il ne reste pas factuel et isolé mais se développe, s'enrichit, se complexifie au contact d'autres savoirs argumentés. C'est un savoir vivant car la réflexion dissertative quand elle est présente invite l'élève à mettre en tension les savoirs les uns avec les autres, à fonder des analogies entre les œuvres, à créer du sens dans le rapprochement des concepts et des exemples ou les différents points de vue sur un même objet. La dissertation peut ainsi apparaître utile car elle cherche à favoriser 


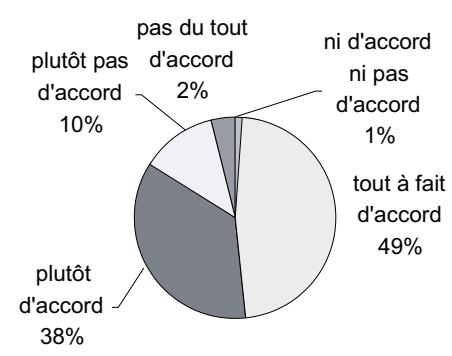

Fig 1 : la dissertation joue un rôle utile dans l'acquisition des savoirs en littérature l'expression d'un discours organisé sur des objets de savoirs, ce qui est aussi un moyen de consolider des acquisitions. Les répondants reconnaissent massivement cette fonction de l'exercice ; ils sont plus de $80 \%$ à être « tout à fait » ou « plutôt d'accord » pour dire que « la dissertation joue un rôle utile dans l'acquisition des savoirs $»$.

Les évolutions de la dissertation témoignent en outre des évo-

lutions de la discipline, elles sont liées à la configuration des savoirs dans la classe de français. Son histoire est dans une certaine mesure une caisse de résonance des ruptures et des réformes que connaît l'enseignement de la littérature dans le second degré. La dissertation est ainsi passée d'un discours à caractère moral ou hagiographique tenu sur les auteurs et les œuvres à un discours moins moralisant mais de portée générale sur la littérature et les faits de culture encourageant alors l'expression de lieux communs et d'une opinion commune sans vrai relief ni singularité. Cette situation encore valable jusque dans les années 80 a sans doute affaibli la valeur formatrice de la dissertation et renforcé son caractère purement rhétorique. S'il est question de problèmes généraux liés à la littérature ou à des débats sur des questions de société, il manque un corpus de références précises, des savoirs objectifs à partir de quoi l'élève peut développer une réflexion. C'est pourquoi on assiste dans les années 90 à un mouvement de recentrage des savoirs et à une réduction du champ des interrogations possibles : des œuvres patrimoniales (Les Confessions, Electre), des questions littéraires (le roman d'apprentissage, maîtres et valet) forment alors un programme d'étude pour les classes d'examen.

Cette évolution des relations dissertation/savoirs est confirmée au moment de la réforme des Programmes de 2001 (MEN, 2001b). La dissertation puise à présent ses problématiques dans un champ de savoirs élargi à des objets d'étude (le biographique, l'épistolaire, la poésie, le théâtre). Ce découpage du programme en grande sections génériques assorties de problématiques restreintes par exemple à la question de la représentation pour le théâtre ou à celle des visions de l'homme et du monde pour le roman consolide la volonté de proposer des savoirs circonscrits, situés dans un environnement déjà problématisé. L'articulation entre un écrit d'évaluation et les savoirs littéraires enseignés sort ainsi renforcée. La critique à l'encontre des champs de savoirs interrogés par la dissertation est aujourd'hui plus difficile à soutenir. Ce qui explique sans doute cet assez large accord autour de la liaison établie entre la dissertation et les objets d'étude : 78\% des répondants jugent favorablement ce rapprochement (« tout à fait» et « plutôt d'accord » cumulés, Fig. 2).

Ces pourcentages corroborent ce qui a été souligné, à sa-

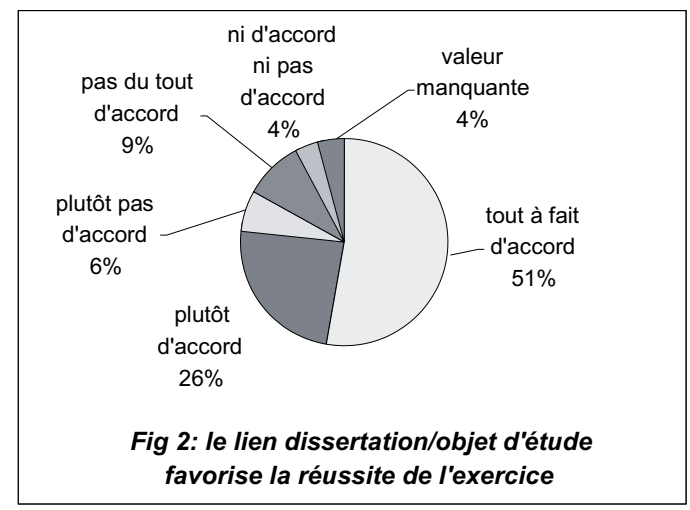


voir que le matériau sur lequel se construit un raisonnement dissertatif s'avère primordial et qu'un enseignement utile de la dissertation ne peut faire l'économie d'un référentiel consistant, clairement identifié. L'élève dispose à présent d'un savoir; il l'a à sa disposition mais ce dernier doit encore être transformé. L'une des difficultés est de ne pas réduire la dissertation à la simple présentation d'un " savoir restitué » et d'opérer la mutation de ce savoir en "savoir questionné ». Ce qui dans un schéma traditionnel de la rhétorique constitue l'étape de l'inventio ne peut être négligé. Elle justifie, je crois, la présence de la dissertation dans un processus d'acquisition des savoirs dans le déroulement du cours de français; elle revêt alors une fonction plus heuristique qu'évaluative. Je reprendrai cette hypothèse dans le chapitre concernant les apprentissages.

Que la dissertation joue un rôle actif dans l'acquisition des savoirs et que le matériau de l'inventio soit emprunté aux savoirs enseignés déclinés en objets d'étude, cela semble bien reconnu par une majorité des professeurs interrogés. En revanche, cet accord devient plus problématique quand il s'agit de savoir si cela concerne tous les élèves et s'adresse indifféremment à l'ensemble des sections en classe de Première, après la Seconde de déter-

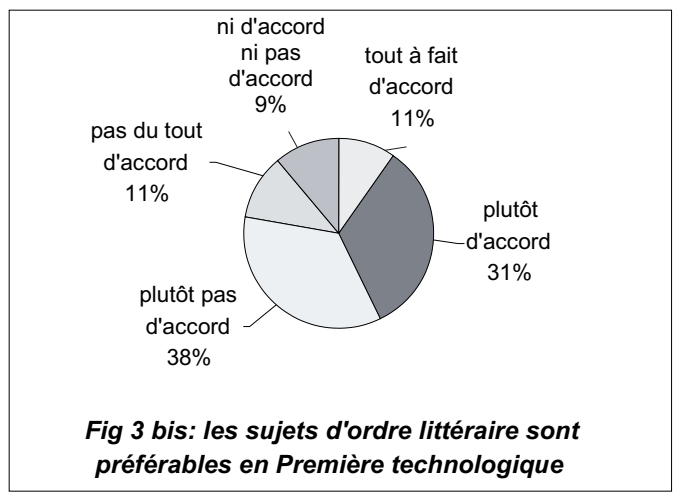
mination. En effet, les items 3 et 3 bis révèlent une opinion contrastée entre les séries générales et technologiques. Si la préférence va aux sujets de nature littéraire

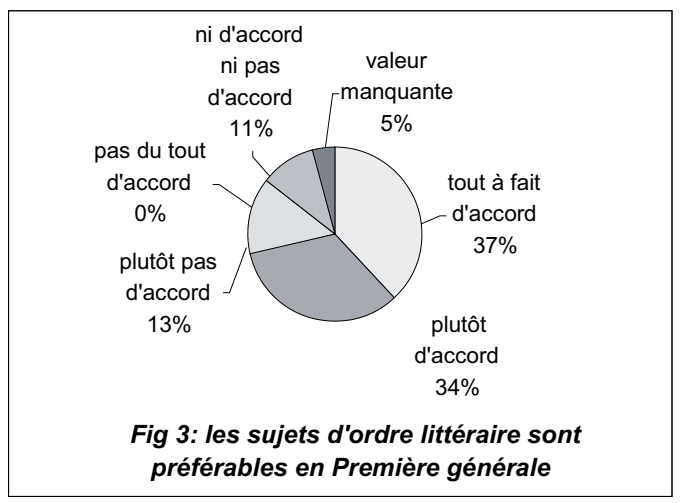
pour les classes de Première L, ES et $\mathrm{S}(71 \%$ en regroupant les valeurs « tout à fait d'accord » et " plutôt d'accord " contre 13\% pour « plutôt pas d'accord»), il n'en est pas de même pour les séries technologiques.

La forte proportion de la valeur manquante $(33 \%)$ s'explique par le fait qu'un certain nombre de professeurs n'enseignent pas dans ces filières. Par ailleurs, $33 \%$ des opinions exprimées se prononcent «plutôt » ou « tout à fait » contre, quand $28 \%$ sont d'un avis contraire. Ajoutons que ce ne sont pas les opinions extrêmes qui l'emportent. Les apprentissages de la dissertation ne vont pas de soi mais cela est encore plus marqué dans les classes des séries technologiques. La faiblesse des savoirs littéraires, le peu de motivation pour les enseignements généraux, les problèmes d'expression à l'écrit sont autant de facteurs qui peuvent expliquer cette différence d'appréciation entre les filières... une différence d'appréciation que l'on retrouve dans l'évaluation des sujets proposés lors des EAF. Je retiendrai ici deux éléments marquants.

Près de $70 \%$ des répondants jugent que les sujets des EAF sont adaptés concernant les Premières générales (Fig. 9). Ce point me semble digne d'être souligné. Il 
tend à valider le dispositif général d'apprentissage de la dissertation en relation avec l'étude des différents domaines littéraires ( $c f$. item 2), et 1'approche problématisée des savoirs ( $c f$. item 7), les sujets EAF ayant depuis 2002 une orientation nettement problématisante. Cette opinion favorable reconnaît une double qualité aux sujets du baccalauréat : celle de se placer convenablement dans la continuité des enseignements et donc de rendre possible une sanction équilibrée des savoirs supposés acquis.

L'autre élément va dans le sens des observations précédentes. On observe une

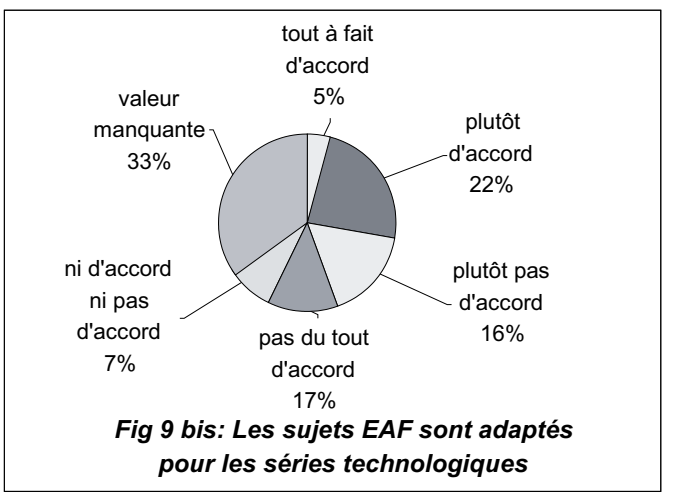
dispersion très marquée des opinions dans l'item 9 bis. En laissant de côté la valeur manquante dont j'ai expliqué par ailleurs l'origine, on constate un net fléchissement des valeurs positives (27\%). L'enseignement de la littérature comme son évaluation tels qu'ils sont conduits actuellement dans les classes des séries technologiques suscitent à l'évidence de sérieuses réserves. Rappelons qu'il n'y a pas si longtemps les sujets de composition française étaient de na-

ture différente suivant les sections ; les élèves de séries technologiques pouvaient composer sur des problèmes généraux (" goût pour la musique, la mode $» . . .($ Charolles, 1990, p. 6)). L'institution a pris une autre option en uniformisant les problématiques. Du point de vue des enseignants, cela ne semble pas satisfaisant.

Les nouvelles mises en contexte de la dissertation dont le signe le plus marquant est la présence d'un corpus de textes littéraires au moment des épreuves du baccalauréat ainsi que l'objectivation des savoirs ont pour conséquence un affaiblissement du critère d'originalité et de sincérité qui était auparavant mis en avant dans l'évaluation de l'exercice (Delforce, 1992, p. 3). A présent que le bagage littéraire est clairement explicité, on ne fait plus de l'originalité et de la singularité de la production une valeur première. Plutôt que la valorisation de la distinction, on attend une conformité dans les références comme dans les argumentaires. Les savoirs d'expérience du lecteur/élève valorisent toujours une production mais ils sont à présent concurrencés voire dominés par des savoirs scolaires et institutionnels. L'important réside dans la manière dont le candidat travaille et pense à partir d'un socle commun de savoirs, dans la manière dont il élabore un raisonnement. Et c'est peut-être à ce prix que la valeur de la dissertation est aujourd' hui restaurée. 


\section{La dissertation, une pratique argumentative à clarifier}

La dissertation interviendrait donc dans l'acquisition des savoirs littéraires. Mais sous quelles formes ces savoirs sont-ils présentés et en réponse à quelle intention discursive? La dissertation est-elle reconnue comme le « parangon de toute argumentation » (Baudart, 1996, p. 80) et ceci renforce-t-il sa valeur en tant que genre scolaire ? Pour une large majorité des répondants, l'exercice de la dissertation entre dans le champ des pratiques argumentatives. Plus de $90 \%$ des professeurs attestent l'existence de ce lien qui signe l'orientation discursive du genre (Fig. 4).

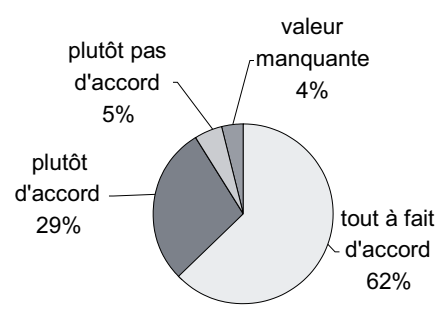

Fig 4: La dissertation permet l'acquisition de techniques argumentatives

La reconnaissance d'une acquisition des procédés et des formes textuelles de l'argumentation à travers l'écriture de la dissertation ne doit pas pour autant nous faire oublier le statut particulier de ce type d'écrit. Michel Charolles (1990, p. 10) distingue ainsi la dissertation des autres discours argumentatifs du fait de son caractère spéculatif : « La dissertation, contrairement aux formes d'argumentation que 1'on vient de citer, n'est pas tournée vers l'action : elle ne vise pas, au moins directement, à influer sur une décision ». Le lecteur/correcteur peut ne pas partager les conclusions d'une dissertation mais apprécier et juger positivement la recevabilité et l'étayage des arguments (Thyrion, 2006) pour peu que ceux-ci ne restent pas dans le strict domaine de la subjectivité mais se réfèrent à des faits littéraires objectifs.

En outre, le lien habituellement établi entre argumentation et opinion personnelle ne va pas non plus de soi. Et si l'on peut parler d' « illusion » à propos de l'expression d'un jugement personnel dans la dissertation (Delforce, 1992, p. 4), c'est bien pour souligner que nous demeurons dans le champ des idées construites, transmises et empruntées et qui sont exposées avec plus ou moins d'éloquence et d'à propos ; qu'enfin l'originalité du jugement ne constitue pas le premier critère de qualité de la dissertation. La formulation adoptée dans les sujets des EAF depuis 2002 me semble à ce propos significative. Les expressions telles que « pour vous », " selon vous » que l'on rencontrait auparavant (Delforce, 1992, p. 3) ont aujourd'hui disparu. La formule la plus souvent retenue, la voici : « Vous répondrez à cette question en un développement composé (ou argumenté) en prenant appui sur les textes du corpus, sur ceux que vous avez étudiés en classe et sur vos lectures personnelles ». L'écriture de la consigne répond à une plus grande demande d'objectivité et insiste sur la convocation des références et des savoirs que les programmes ont délimités.

Ainsi, entre l'affaiblissement de l'opinion et de l'exercice de la conviction, on pourrait penser que la dissertation se vide de sa substance argumentative (Darras et alii, 1994) et que ce qui est premier, c'est la présence d'un raisonnement convenablement étayé. Faire la preuve de ses connaissances tout en les situant dans un questionnement ; l'exigence de restitution d'un savoir se double de l'adoption d'une posture interrogative. C'est cette combinaison qui constitue l'enjeu de la dissertation, le pari dissertatif. Et si, comme le souligne Bernard Delforce, la dissertation est bien « l'expression d'une opinion mais qui se présente comme adop- 


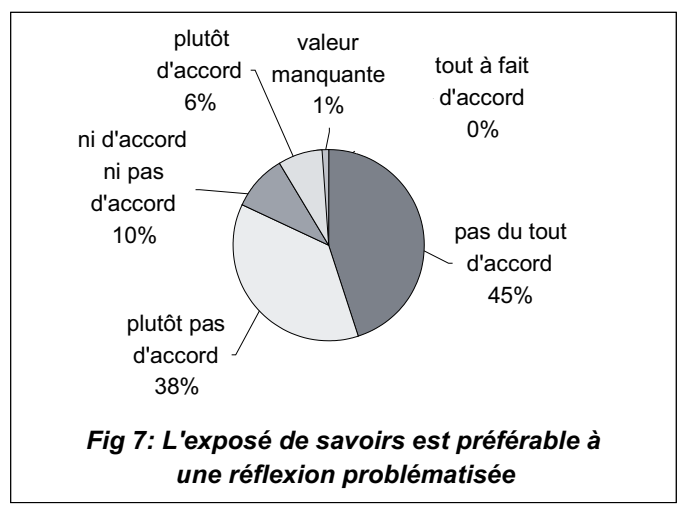
au raisonnement sous la forme d'une réponse argumentée à une question est validée par une majorité de professeurs (Fig. 7).

Le pourcentage cumulé des opinions «pas du tout » et "plutôt pas d'accord» (82\%) ainsi que la non représentation d'opinion «tout à fait d'accord » rappellent fermement la spécificité de la dissertation dans la production des savoirs. Il s'agit pour le scripteur de mobiliser des savoirs en vue de circonscrire un problème, d'en examiner les différentes facettes et non de s'en tenir à une compilation de connaissances sur un thème littéraire. Convenons toutefois que ce programme dissertatif appelle quelques nuances. Une lecture un peu attentive des sujets des EAF montre en effet des degrés d'argumentativité (1) (Masseron, 1997) dans la manière dont sont posés les problèmes. Je retiendrai quatre degrés, un nombre suffisant pour représenter les variations dans l'exposition de la problématique :

- Un degré faible : une question ou un domaine littéraire est soumis à l'examen du scripteur ; cet examen relève plus de la description que de l'expression d'un débat. Le scripteur doit par exemple présenter les différentes propriétés d'un champ littéraire.

Deux exemples : «Romans policiers, romans criminels : comment expliquez-vous que tant de lecteurs prennent plaisir à ce genre de récits où règnent pourtant par convention le mal, la violence et la mort ? Vous répondrez à cette question de façon organisée et argumentée en vous référant à des exemples précis tirés des textes du corpus, d'œuvres étudiées en classe et de vos lectures personnelles. » (section STT, centres étrangers, session 2009); «En vous appuyant sur le corpus, vos lectures et éventuellement votre expérience de spectateur, vous vous demanderez de quelles ressources spécifiques dispose le théâtre pour représenter les conflits, les débats, les affrontements qui peuvent exister dans les rapports humains. » (section L, centres étrangers, session 2006).

- Un degré moyen : le sujet propose l'examen d'une question littéraire dont on signale le caractère incomplet par l'emploi d'une formule restrictive ; une propriété est validée mais il est aussitôt précisé que celle-ci ne rend compte que d'une partie de l'objet considéré.

Un exemple : «Le but d'une dernière page est-il uniquement de donner un dénouement à l'histoire? Vous répondrez dans un développement organisé, en vous ap-

(1) Caroline Masseron énumère un certain nombre de paramètres qui permettent de mesurer l'argumentativité d'un discours et de reconnaître le caractère graduable de la notion : « explicitation des connexions d'argument à conclusion; axiologisation marquée ; attestation d'une intention argumentative directe... » p. 20. 
puyant sur les textes du corpus, les œuvres étudiées en classe et vos lectures personnelles. » (sections L, ES, S, centres étrangers, session 2009).

— Un degré accentué : il s'agit d'examiner une question littéraire dont la formulation repose sur un paradoxe, une apparente contradiction, un décrochage avec le sens commun.

Un exemple : «Le souci de vérité dans l'écriture autobiographique interdit-il mise en scène, détour, masques littéraires ? (sections L, ES, S, centres étrangers, session 2007).

- Un degré intense : 1'examen de la question suppose des prises de position contradictoires, repose sur le jeu ouvert de l'alternative et du débat.

Un exemple : «La rébellion contre l'héritage des poètes précédents est-elle indispensable à la création poétique ? Vous répondrez en vous appuyant sur les textes qui vous sont proposés, ceux que vous avez étudiés en classe et vos lectures personnelles. »(sections S, ES, session 2005).

La problématique, qu'elle fasse l'objet d'un guidage plus ou moins marqué, d'une orientation plus ou moins accompagnée par les termes et la forme du libellé, demeure une composante essentielle dans l'art de raisonner. Par le jeu du questionnement, elle est censée encourager l'évaluation d'un savoir littéraire. La littérature est porteuse de valeurs, elle fait l'objet de jugements esthétiques, éthiques, elle propose des formes d'expression variables, inscrites dans une histoire. En ce sens, elle est de plain pied dans l'espace du négociable, du révisable (Veck, 1997) et c'est aussi cette vision que la dissertation avec le concours de la problématique défend et promeut.

La problématique est, idéalement, une mise en dialogue de savoirs. Elle est aussi l'axe autour duquel ces savoirs sont distribués ; en ce sens elle est à la jonction entre les étapes rhétoriques de l'inventio et de la dispositio et assure leur coordination. En fonction du degré d'argumentativité présent dans le libellé du sujet, de l'angle d'ouverture du débat, un guidage plus ou moins fort se met en place. Dans le premier sujet des EAF précédemment cité (session 2006, section L), il est clair que l'objectif est moins de discuter la capacité du théâtre à exposer des conflits que de réfléchir au comment de cette exposition. Al'inverse, on considérera que le dernier sujet cité consacré à la question de l'héritage poétique laisse une plus grande responsabilité au scripteur puisqu'il doit avancer une opinion recevable au terme de l'examen des hypothèses contradictoires.

Ce dernier type de raisonnement appelé polémique et que la tradition scolaire reconnaît dans la (trop) fameuse «thèse-antithèse-synthèse » est-il le mode dissertatif que le professeur doit nécessairement aborder dans les apprentissages?

Les réponses à cet item font certes apparaître un rejet du caractère indispensable du plan dialectique mais pas de façon massive $(55 \%)$. Les opinions sont moins tranchées et rendent compte d'expériences professionnelles certainement très diverses. Comme nous l'avons vu, la démarche réflexive et problémati-

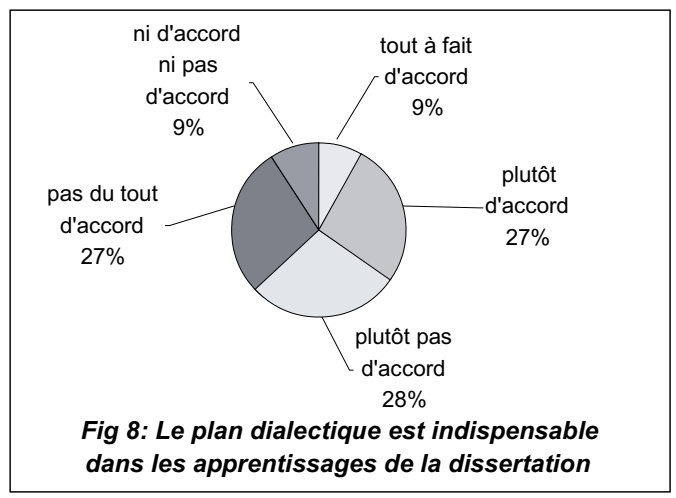


que est fortement défendue mais cela peut s'accompagner d'une plus grande souplesse du point de vue formel. La pratique polémique peut s'exercer différemment et comme le souligne Bernard Delforce « les sujets, même quand ils ne sont pas de nature polémique et n'empruntent pas le schéma du même nom, se prêtent à des moments polémiques » (Delforce, 1992, p. 16). L'imposition d'une forme, parce qu'elle tend à rigidifier l'exercice et à en accentuer l'artifice (Darras, 1991) masque aussi les enjeux plus déterminants de la formation à la dissertation dont nous allons voir à présent certaines de ses caractéristiques.

\section{Vers un apprentissage renouvelé de la dissertation?}

Dans cette dernière partie de l'analyse des discours, seront présentées brièvement les difficultés d'apprentissage qui sont je crois bien connues. Les démarches de formation décrites dans les questionnaires me donneront par ailleurs l'occasion d'avancer quelques propositions susceptibles d'élargir les modes d'appropriation d'un exercice réputé difficile.

\section{Un apprentissage difficile}

La formation à la dissertation n'est pas chose aisée. Elle suppose la mobilisation de savoirs complexes ainsi que des compétences cognitives et langagières élaborées, difficiles à mettre en

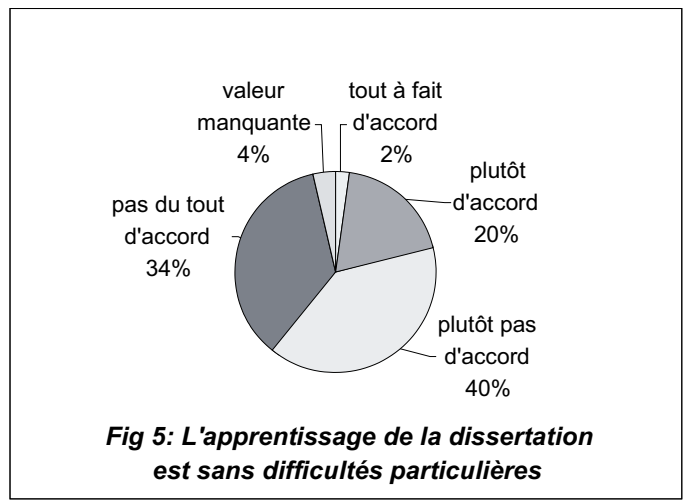
œuvre au cours de deux années déjà très chargées. C'est le sentiment dominant qui ressort des réponses à 1 'item 5 , avec un écart important entre les valeurs positives et négatives $(22 \%$ contre $74 \%$ ). La reconnaissance de la difficulté de l'apprentissage trouve un écho dans les réponses à l'item 6. L'hypothèse d'une bonne connaissance de la dissertation par les élèves en fin de classe de Première n'est admise que par $32 \%$ des répondants (valeurs « tout à fait » et «plutôt d'accord » cumulées), alors qu'ils sont $60 \%$ à penser le contraire.

Le temps de l'inventio

Au chapitre des difficultés rencontrées par les élèves (item 15), les professeurs notent en premier lieu et de façon insistante le manque de savoirs littéraires, le peu de références susceptibles de constituer des exemples adaptés. Ces lacunes sont jugées particulièrement dommageables, la pauvreté du matériau réduisant 1 'exercice à 1'énoncé de quelques arguments juxtaposés. Ce constat excède le

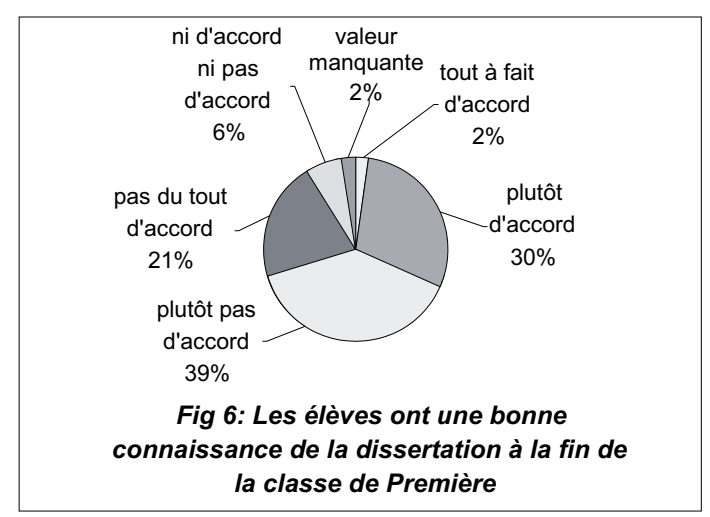


cadre immédiat de l'apprentissage de la dissertation et interroge les modes d'acquisition des savoirs en classe de français ; mais il est un autre point qui revient fréquemment : c'est la difficulté de l'élève à faire du lien, à exploiter le savoir et les références littéraires développés dans le cadre des séquences consacrées aux objets d'étude. De même est soulignée la difficulté à tirer parti des corpus qui accompagnent normalement les sujets, sur le modèle de ce qui est proposé aux EAF. Le savoir dont peut disposer l'élève est souvent un savoir inactivé, les extraits qui forment les corpus restent muets au sens où l'élève ne fait pas les inférences qui lui permettraient de construire un savoir accordé à la situation argumentative particulière définie par la problématique. On reconnaît notamment ici aussi le défaut d'une démarche de conceptualisation que G. Vigner divise en deux grandes opérations :

«- une opération de généralisation qui consiste à rassembler plusieurs objets singuliers en une classe unique et à en retenir les caractères communs ;

- une opération de discrimination qui consiste à isoler certains traits à partir d'un répertoire de catégories données. » (Vigner, 1990, p. 25)

Le deuxième domaine dans lequel l'élève se trouve pénalisé concerne la lecture du sujet et l'expression pertinente de la problématique. Cette phase de l'élaboration de la dissertation est souvent décrite dans les manuels de méthodologie comme le temps de l'examen lexical et syntaxique du sujet (Thyrion, 1996, p. 18 et $s q$. ; Colson, 1996, p. $11 s q$.). Les questionnaires font état de ces étapes (item 13); il est régulièrement question de « l'analyse du sujet», de « la recherche des présupposés et paradoxes implicites», "des couples d'opposition», de la «définition des termes ». On rappellera toutefois que l'analyse sémantique et formelle du sujet est souvent insuffisante pour décrire un sujet et dégager une problématique et que la réflexion doit s'appuyer aussi sur des éléments externes ; ce que souligne précisément B. Delforce dans le commentaire suivant :

[La problématique] signale le lieu d'un vrai problème, mais elle ne le formule pas de façon adéquate. On l'obscurcit, en effet, en invitant les élèves à chercher la problématique dans le sujet tel qu'il est formulé, alors qu'elle se situe d'abord en dehors de lui, dans l'espace de questionnement qui l'englobe. Le sujet est moins une série de questions qui y seraient cachées et qu'il faudrait découvrir par une analyse de mots, qu'une réponse qui fait question, qui appelle ou soulève des questions qu'il faut examiner. Le sujet sur lequel on invite à réfléchir n'est, en fait, qu'une des réponses possibles à une question qui, le plus souvent, n'est pas formulée. (Delforce, 1992, p. 13).

Les auteurs de Apprentissages de la dissertation $3 \% 2^{\circ}$ exposent justement un ensemble de démarches qui offrent l'intérêt de donner des outils à l'élève en même temps qu'elles visent la compréhension d'un sujet à partir d'activités cognitives diversifiées (Darras et alii, 1994, pp. 79-136). La confrontation de sujets, la production de sujets, les mises en relation entre le sujet et le corpus, les reformulations, la mise au jour des présupposés forment une panoplie précieuse qui trouve avantage à être intégrée à plusieurs moments d'une séquence consacrée à un objet littéraire. Cette question de la temporalité des apprentissages n'est en effet pas abordée dans les questionnaires, pas plus que dans les ouvrages et manuels méthodologiques qui s'intéressent à la dissertation. Elle constitue pourtant une voie intéressante.

L'exercice est généralement travaillé dans la perspective de la préparation aux EAF ; il s'agit alors d'acquérir une méthodologie ad hoc que l'élève pourra exploi- 
ter le jour de l'examen. L'analyse des réponses à l'item 13 montre que la démarche fondée sur les étapes de la rhétorique traditionnelle est ici largement privilégiée. Cette décomposition des apprentissages, on la rencontre dans nombre d'ouvrages consacrés à l'apprentissage de cet écrit à destination des classes de lycée comme des premiers cycles universitaires (Scheiber, 1989; Preiss, 1989; Thyrion, 1996 ; Colson, 1996 ; Adam, 1999). L'objectif est de construire une méthodologie qui prend ensuite la forme d'une fiche outil : c'est autour de cette fiche outil que s'organisent les apprentissages. L'apprentissage de la dissertation est positionné à des moments spécifiques (méthodologie des épreuves de l'EAF); sa mise en œuvre est effective en fin de séquence au moment de l'évaluation d'un objet d'étude.

Cette organisation n'est cependant pas incompatible avec une démarche qui met plus nettement en relation les pratiques de raisonnement dissertatif avec les savoirs littéraires dans le cours même de leurs acquisitions. En somme, il s'agit de conférer à la dissertation une fonction qui ne soit plus seulement évaluative, en l'introduisant dès le début d'une séquence littéraire. Que le libellé d'une dissertation puisse devenir la question qui ouvre une séquence et alors la dissertation est en mesure de jouer une fonction heuristique d'investigation et de construction des savoirs. Cette réorientation de la dissertation est une réponse parmi d'autres aux difficultés constatées dans les phases de l'inventio. En proposant une problématique, support d'une dissertation, en ouverture de la séquence, on multiplie les occasions de mettre en rapport les savoirs et la question à différentes étapes du cours ; on peut demander aux élèves d'établir des liens réguliers entre le questionnement dissertatif et les savoirs discutés au cours des lectures. Cela doit permettre de mieux comprendre comment une dissertation peut être alimentée par des savoirs. L' inventio s'élabore dans le cours de la séquence, la formation à la dissertation s'inscrit dans une durée qui retient l'importance de la dimension formative de l'évaluation.

\section{Le temps de la dispositio}

Une proportion importante de questionnaires relève les difficultés relatives à la mise en texte. Les insuffisances concernent l'organisation des idées, la hiérarchisation des arguments, la structure générale de l'argumentation. Alors que la description des apprentissages (item 13) privilégie le plus souvent l'approche de la dissertation sous sa forme rhétorique conventionnelle et traite de l'écriture de l'introduction, de la conclusion et du paragraphe argumentatif, il semble opportun d'examiner les points de contact possibles avec les autres formes de discours. Comprendre les principes d'organisation ainsi que les règles d'énonciation de la dissertation peut se faire dans le cadre d'une démarche comparative. Les manuels de méthodologie ont le défaut de présenter les types d'écrits de façon cloisonnée. Or, la compréhension des contraintes formelles et énonciatives de la dissertation peut aussi et de façon profitable être mise en place à partir d'une pratique active de réécriture et de transformation des discours. La transposition d'un écrit d'invention métatextuel du type de la lettre ou de l'article en tout ou partie d'une dissertation constitue un apprentissage formateur en ce sens qu'il permet d'aborder des écrits d'évaluation présents au baccalauréat dans leurs singularités et leurs caractéristiques communes. Outre les aménagements structurels que cela suppose liés au genre de discours, on peut aussi travailler les aménagements aussi essentiels qui relèvent de l'énonciation. L'apprentissage ne se développe pas alors à partir d'une fiche méthodologique qu'il s'agirait d'appliquer mais retient plutôt des phases de découverte et de manipulation. Une pratique de la variation discursive même fragmentaire et limitée peut être retenue comme une initiation active en vue 
de l'acquisition et la compréhension des codes des discours scolaires, qu'il s'agisse de la dissertation, de l'écrit d'invention et du commentaire littéraire.

\section{Le temps de 1'elocutio}

La rédaction de la dissertation est une étape individuelle contrairement aux étapes précédentes généralement envisagées de façon collective dans l'apprentissage. L'écriture est l'étape ultime, la mise en texte d'une argumentation déjà élaborée. Faute de temps, on lui accorde finalement peu d'attention en classe et la remédiation est surtout envisagée dans une perspective de correction de la langue et de mise en conformité des règles de production. Or, le fait d'inscrire les apprentissages dans une autre temporalité, de mettre les élèves en situation de produire de façon régulière dans le cours de la séquence peut rétablir une fonction heuristique de l'écriture comme lieu où les savoirs aussi s'élaborent progressivement. De même aurait-on intérêt à favoriser la réécriture, même partielle, des productions afin de réduire la distance entre l'élève et l'écriture réflexive... mais c'est encore un autre chantier.

\section{Conclusion}

Je me suis efforcé tout au long de cet article d'exposer les motifs d'une large reconnaissance de l'exercice de la dissertation par le professeur de français, et ce sans distinction de génération, d'expérience professionnelle en lycée ou encore de grade.

Cette reconnaissance, qui par certains aspects prend l'allure d'une défense d'un élément déterminant de la discipline « français » au lycée, est apparue évidente à différentes étapes du questionnaire. La mise en perspective des différents items, le croisement des différents informations dessinent le profil cohérent d'un exercice qui remplit sa fonction de codification des savoirs littéraires en classe de français. La prégnance du référentiel contenu dans les objets d'étude assure à la dissertation une position pourrait-on dire d'autorité pour mesurer l'acquisition des connaissances sous la forme d'un écrit restitutif dont les règles sont bien connues et partagées par une majorité de professeurs.

La stabilité de l'exercice, la reconnaissance des étapes traditionnelles de la pratique rhétorique dans le cadre de l'apprentissage, les liens objectifs entre les objets d'étude et les problématiques des sujets EAF assoient une légitimité que la dissertation partage sans doute plus avec le commentaire littéraire qu'avec l'écrit d'invention.

A côté de l'intérêt porté à la dissertation, les réponses au questionnaire ont aussi mis en évidence les difficultés de son apprentissage. Comme activité conceptuelle tout d'abord, les réponses insistent sur la difficulté pour l'élève à opérer des rapprochements pertinents entre un questionnement proposé par un sujet et un corpus littéraire, à conduire une sélection et une transformation adéquates des savoirs comme réponse possible à une question posée. Comme pratique langagière ensuite, les questionnaires font état des problèmes inhérents à la production d'un discours argumenté mais mettent toutefois l'accent sur les carences liées à la mise en cohérence du développement dissertatif. La gestion complexe de l'observation d'un fait ou d'un problème, son explicitation et son illustration, l'expression d'une opinion qui, toutes, contribuent à la progression de la réflexion jusqu'à son terme, est difficilement maîtrisée par l'élève qui présente par ailleurs des déficits dans la maîtrise de la langue. Les différences d'appréciation entre les séries générales et technologiques me paraissent à ce titre révélatrices des défis posés à un enseigne- 
ment de la littérature auprès d'élèves réputés faibles lecteurs, faibles scripteurs. Peut-être quelques éléments de réponse ou du moins des pistes de travail sont à chercher, moins du côté de la prescription d'une fiche méthodologique et de l'application d'un modèle que du côté de la mise en place d'un parcours épistémologique de l'exercice de la dissertation.

En effet, il me semble qu'une connaissance pratique de l'exercice a des chances de se réaliser en tirant parti de l'environnement dans lequel l'apprentissage est mis en place. Un environnement discursif tout d'abord qui permet de réfléchir concrètement, par l'exercice de la manipulation et de la transposition, aux fonctionnements respectifs de la dissertation et de l'écrit d'invention à vocation argumentative. Un environnement planifié des savoirs et des pratiques littéraires (lecture/écriture) qui peut inciter l'enseignant à introduire plus tôt dans sa séquence la dissertation et renforcer ainsi son rôle d'investigation. La dissertation comme objet didactique mérite assurément notre attention...

\section{Références}

ADAM, F. (1999) : Réussir la dissertation littéraire, Paris, Dunod.

BAudart, F., CARIOU, D., FAURE, M.-F. (1998) : « Les demandes de formation sur l'argumentation en collège et en lycée ", Le Français aujourd'hui n ${ }^{\circ} 123$, pp. 8090.

Charolles, M. (1990) : «La dissertation quand même », Pratiques n 68, pp. 5-16.

Colson, J. (1996) : Le dissertoire, Bruxelles, De Boeck Université.

DARRAS, F. (1991) : «Les avatars du plan dialectique : une aporie didactique », Recherches $\mathrm{n}^{\circ} 14$, pp. 61-74.

Darras, F., DAunay, B., Delcambre, I., VAnSEVERen, M.-P. (1994) : Apprentissages de la dissertation $3^{e} / 2^{e}$, CRDP de Lille.

DARRAS, F., DELCAMBRE, I. (1992) : «Des modules d'apprentissage du genre dissertatif $»$, Pratiques $\mathrm{n}^{\circ} 75$, pp. 17-43.

DelCAmbre, I. (1990) : « De l'argumentation à la dissertation. Analyse d'une démarche d'apprentissage », Pratiques ${ }^{\circ}$ 68, pp. 69-88.

- (1996) : «Construire une séquence didactique autour d'un problème d'écriture : 1'exemplification », Pratiques $n^{\circ}$ 92, pp. 57-82.

DELFORCE, B. (1985) : «Approches didactiques de la production d'un écrit "fonctionnel" : les difficultés de la dissertation », Pratiques $n^{\circ} 48$, pp. 35-52.

- (1992) : «La dissertation et la recherche des idées ou : le retour de l'inventio», Pratiques $\mathrm{n}^{\circ} 75$, pp. 3-16. 
Genette, G. (1969) : « Rhétorique et enseignement », in Figures II, Paris, Seuil.

HALTÉ, J.-F. (1989) : «Analyse de l'exercice dit "la rédaction" et propositions pour une autre pédagogie de l'écriture », in M. ChAROLLES, J.-F. HALTÉ \& C. MASSERON (Éds.), Pour une didactique de l'écriture, pp. 9-47. Metz: Centre d'Analyse Syntaxique de l'Université de Metz.

HoudART-MEROT, V. (1998) : La culture littéraire au lycée depuis 1880. Rennes, P. U. Rennes.

JEY, M. (1998) : La Littérature au lycée : invention d'une discipline (1880-1925). Metz, Centre d'Études Linguistiques des Textes et des Discours.

LE GOFF, F. (2005) : «L'écriture d'invention : ce que disent les professeurs de leurs pratiques », Pratiques n $127-128$, pp. 60-74.

MASSERON, C. (1997) : «Pour une didactique de l'argumentation (écrite) : problèmes, objets, propositions », Pratiques n ${ }^{\circ}$ 96, pp. . 7-34.

MinistĖre DE L'ÉDUCATION NATIONALE. (MÉN, 2001a). Bulletin Officiel, nº 26 du 28 juin.

— (MÉN, 2001b). Bulletin Officiel, n²8 du 12 juillet 2001.

PREISS, A (1989) : La dissertation littéraire, Paris, Armand Colin.

SCHEIBER, C. (1989) : La dissertation littéraire, Paris, Bordas.

THYRION, F. (1990/96) : La dissertation, du lieu commun au texte de réflexion personnelle, Paris, Duculot.

— (2006) : «Argumenter par écrit : quelle didactique ? ", Namur, Diptyque n ${ }^{\circ 5}$, pp. 55-80.

Vigner, G. (1990) : «Argumenter et disserter : parcours d'une écriture », Pratiques $\mathrm{n}^{\circ} 68$, pp. 17-56.

VECK, B. (1990) : «Penser/classer. Une étude des procédures de classement et de constitution des faits dans les copies de composition française au lycée », Pratiques n68, pp. 57-67.

— (1997) : «Problématiser en littérature », in La problématique d'une discipline à l'autre, ADAPT, pp. 113-120.

VialA, A. (1990) : « Table ronde, la dissertation : Naissance et évolution de l'exercice scolaire ", Pratiques n ${ }^{\circ} 68,1990$, pp. 108-109.

Wlassoff, M. (1998) : «La dissertation française vue de Pologne », Le Français aujourd'hui $\mathrm{n}^{\circ} 123$, pp. 91-95. 


\section{ANNEXE \\ Questionnaire remis au professeur}

\section{La dissertation au lycée : rôles, apprentissage, évaluation.}

Informations générales :

- Nombre d'années d'enseignement au lycée :

- Age :

- Statut : non titulaire, Plc 2, certifié(e), agrégé(e)

- Qualité du lycée : enseignement général ; général et technologique ; agricole

- Implantation du lycée : banlieue ; ville, sep, zone sensible, zone rurale

- Présence de classes d'enseignement supérieur dans le lycée (BTS, classes préparatoires) : oui, non

- Département :

Les réponses aux premiers items se font selon le principe suivant :

$\square$ Tout à fait d'accord $\square$ Plutôt d'accord

$\square$ Plutôt pas d'accord $\square$ Pas du tout d'accord $\square$ Ni d'accord, ni pas d'accord

Des compléments d'information et de réflexion peuvent être apportés pour chacune des rubriques

Il est aussi possible de porter des informations plus développées sur une feuille libre.

1. L'exercice de la dissertation joue un rôle utile dans l'acquisition des savoirs en littérature

2. Le lien explicite entre la dissertation et un objet d'étude permet à l'élève de mieux réussir l'exercice

3. La dissertation sur des sujets d'ordre littéraire est préférable à d'autres types de sujets de discussion

- de Première générale ; - de Première technologique

4. L'apprentissage de la dissertation permet l'acquisition des techniques de l'argumentation

5. L'apprentissage de la dissertation ne présente pas de difficultés particulières

6. La technique de la dissertation est bien connue des élèves à la fin de la Première

7. Il est préférable de proposer des sujets qui appellent un exposé de savoirs plutôt qu'une réflexion problématisée

8. Le plan type «thèse-antithèse-synthèse » est indispensable dans les apprentissages de la dissertation

9. Les sujets de dissertation proposés aux EAF sont adaptés aux élèves. - de Première générale ; - de Première technologique

10. La dissertation pourrait être supprimée de la classe de français et des EAF

11. Quelle place occupe la dissertation dans votre enseignement du français au lycée ? Une place essentielle, importante, suffisante, secondaire?

12. Quels sont les supports que vous utilisez pour former les élèves à la dissertation? (Manuels de la classe, annales, fiches méthodologiques, documents Internet, documents personnels, etc.)

13. Pouvez-vous en quelques lignes présenter la démarche que vous adoptez habituellement pour l'apprentissage de la dissertation?

14. A quels aspects, à quels constituants êtes-vous particulièrement attentif pour juger de la qualité d'une dissertation?

15. Selon vous, quels sont les principaux obstacles rencontrés par les élèves dans la réalisation de l'exercice?

16. Selon vous, quels sont les principales difficultés rencontrées par un enseignant dans la mise en place d'un apprentissage de la dissertation? 